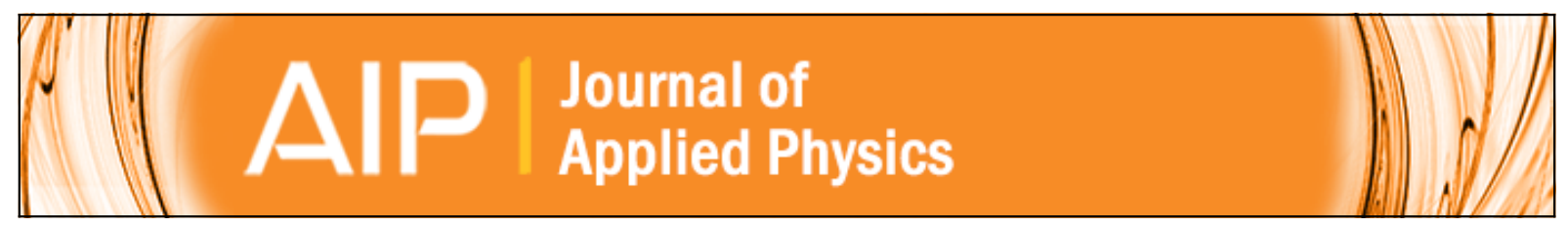

\title{
Magnetic field effect for cellulose nanofiber alignment
}

Jaehwan Kim, Yi Chen, Kwang-Sun Kang, Young-Bin Park, and Mark Schwartz

Citation: Journal of Applied Physics 104, 096104 (2008); doi: 10.1063/1.3006140

View online: http://dx.doi.org/10.1063/1.3006140

View Table of Contents: http://scitation.aip.org/content/aip/journal/jap/104/9?ver=pdfcov

Published by the AIP Publishing

\section{Articles you may be interested in}

Structural and magnetic properties of chemically synthesized Fe doped $\mathrm{ZnO}$

J. Appl. Phys. 105, 07C520 (2009); 10.1063/1.3073933

Nanocrystalline iron nitride films with perpendicular magnetic anisotropy

Appl. Phys. Lett. 92, 052504 (2008); 10.1063/1.2840185

Tuning the crystal structure and magnetic properties of Fe doped In 203 nanocrystals Appl. Phys. Lett. 91, 262503 (2007); 10.1063/1.2815661

Synthesis and characterization of erbia doped metal oxide nanofibers for applications in thermophotovoltaics J. Vac. Sci. Technol. A 25, 922 (2007); 10.1116/1.2742390

Fabrication of $\mathrm{Mn}$-doped $\mathrm{ZnO}$ diluted magnetic semiconductor nanostructures by chemical vapor deposition J. Appl. Phys. 99, 08M119 (2006); 10.1063/1.2173235

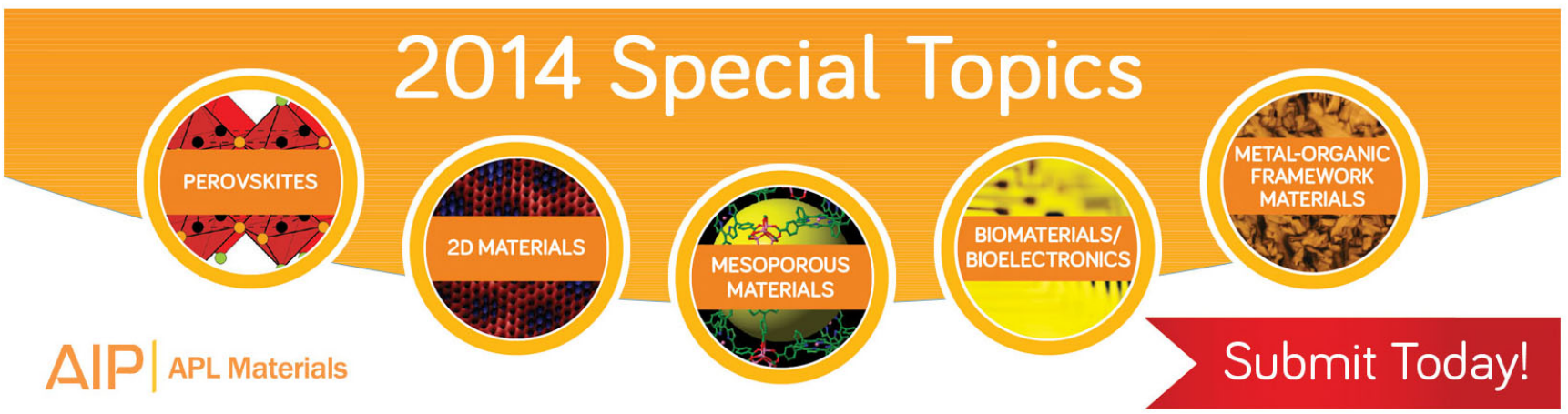




\title{
Magnetic field effect for cellulose nanofiber alignment
}

\author{
Jaehwan Kim, ${ }^{1, a)}$ Yi Chen, ${ }^{1}$ Kwang-Sun Kang, ${ }^{1}$ Young-Bin Park, ${ }^{2}$ and Mark Schwartz ${ }^{2}$ \\ ${ }^{1}$ Creative Research Center for EAPap Actuator, Mechanical Engineering Department, \\ Inha University, 253 Yonghyun-Dong Nam-Ku, Incheon 402-751, South Korea \\ ${ }^{2}$ Department of Industrial and Manufacturing Engineering, Florida AM University \\ and College of Engineering, Florida State University, 2525 Pottsdamer Street, Tallahassee, \\ Florida 32310-6046, USA
}

(Received 7 December 2007; accepted 11 September 2008; published online 7 November 2008)

Regenerated cellulose formed into cellulose nanofibers under strong magnetic field and aligned perpendicularly to the magnetic field. Well-aligned microfibrils were found as the exposure time of the magnetic field increased. Better alignment and more crystalline structure of the cellulose resulted in the increased decomposition temperature of the material. X-ray crystallograms showed that crystallinity index of the cellulose increased as the exposure time of the magnetic field increased.

(c) 2008 American Institute of Physics. [DOI: 10.1063/1.3006140]

Cellulose and its derivatives are one of the most naturally abundant biomacromolecule and among the most widely employed natural polymers in numerous industrial fields, such as the textile industry, ${ }^{1}$ packaging materials, ${ }^{2}$ photographic films, ${ }^{3}$ pharmaceutical membranes, ${ }^{4}$ and thermoplastics. ${ }^{5}$ Compared to conventional use, fabrication of micromold, cellulose fiber composites, and aligned fiber has been extensively studied in application fields including electronic paper $^{6}$ and reinforcement. ${ }^{7-9}$ Recently, regenerated cellulose has been revisited as a smart material for utilizing as sensors and actuators. ${ }^{10-12}$ This smart material renamed as electroactive paper (EAPap) has the advantages of lightweight, low cost, biodegradability, and excellent actuation properties, such as large deformation, low actuation voltage, and low power consumption.

High stiffness and strength of nanoscale natural cellulose fibers are applicable to cellulosic reinforcements resulting to enhancements in high strength and elastic moduli. ${ }^{13}$ Wet drawing of the cellulose film results in reorientation of cellulose microfibrils. The reorientation of cellulose films improves tensile strength and modulus of elasticity. ${ }^{14}$ Meltblown cellulose films from $\mathrm{N}$-methylmorpholine- $\mathrm{N}$-oxide solution have $300 \mathrm{MPa}$ of tensile strength and $8 \mathrm{GPa}$ of modulus of elasticity depending on the direction of the orientation. ${ }^{15}$ Recently, magnetic field effect on the diamagnetic materials has been paid much attention for use in magnetic field on the processing of diamagnetic materials such as organic, inorganic, and polymeric materials. Alignment of the cellulose microfibrils by strong magnetic field $(7 \mathrm{~T})$ was reported by Sugiyama et al. ${ }^{16}$ The aligned structure was also achieved in cellulose nanocrystals, ${ }^{17}$ tunicate cellulose microfibrils, ${ }^{18}$ and cellulose triacetate ${ }^{19}$ by strong magnetic field.

In this paper, we report strong magnetic field effects on the curing process of regenerated cellulose. Field emission scanning electron microscope (FESEM) images were taken on the surface and cross-sectional images of the regenerated cellulose cured under various durations of exposure to mag-

\footnotetext{
${ }^{a)}$ Author to whom correspondence should be addressed. Electronic mail: jaehwan@inha.ac.kr.
}

netic field. Thermogalvanometric analysis (TGA) and x-ray crystallogram were taken for comparison of decomposition temperature and observation of nanofiber structure formation.

The cotton pulp (buckeye) having a high degree of polymerization (4500) was torn into pieces and dried with $\mathrm{LiCl}$ in a heating oven at $100{ }^{\circ} \mathrm{C}$. The cotton pulp was mixed with $\mathrm{LiCl}$ and anhydrous dimethylacetamide and was dissolved by heating at $155{ }^{\circ} \mathrm{C}$ with stirring for $24 \mathrm{~h}$. The solution was cooled to room temperature and stored for one week. The cellulose solution was spin coated onto the silicon wafer. A transparent and smooth cellulose film was obtained.

The magnetic field was applied to the samples using a 7 $\mathrm{T}$ horizontal superconducting magnet (Oxford Instruments) as shown in Fig. 1, which is located at the National High Magnetic Field Laboratory, Tallahassee, FL. The magnet is characterized by a $150-\mathrm{mm}$-diameter through bore. The instrument was controlled such that the magnetic field was regulated at 5.3 T, and the region of magnetic field homogeneity (in terms of magnitude) with $\pm 5 \%$ error was approximately $12 \mathrm{~cm}$ in the axial direction. The sample was placed in the central location of the bore to ensure homogeneous applied field.

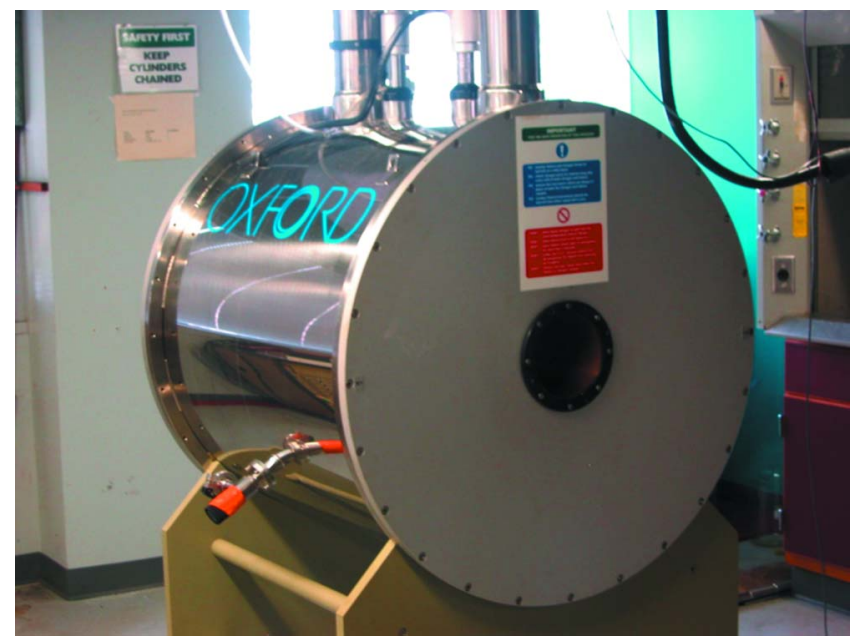

FIG. 1. (Color online) Photograph of $7 \mathrm{~T}$ horizontal superconducting magnet with $150 \mathrm{~mm}$ diameter bore. 


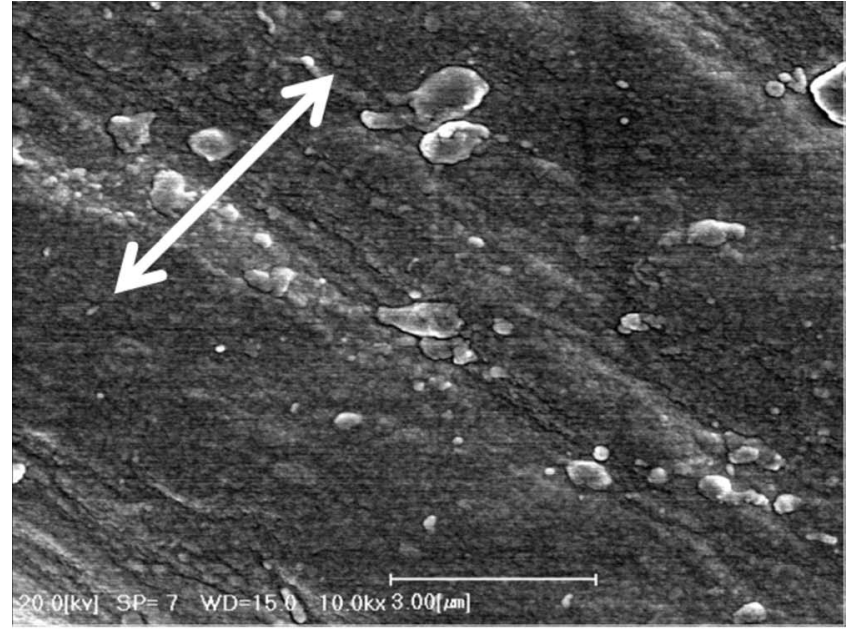

(a)

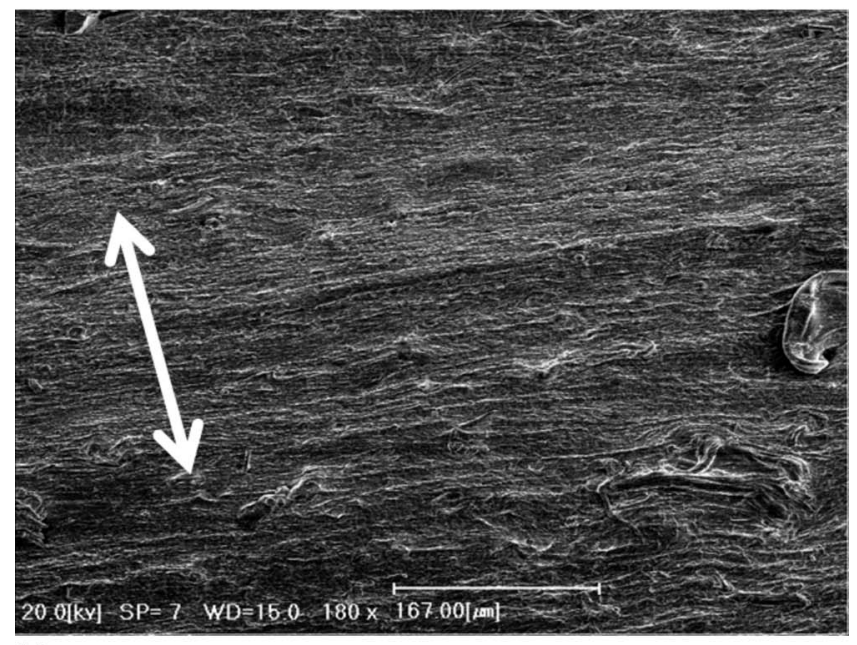

(b)

FIG. 2. FESEM surface images of samples for (a) 60 and (b) $180 \mathrm{~min}$ exposure time of magnetic field.

The cellulose films polled with magnetic field were analyzed using a thin film x-ray diffractometer (X'pert MPD, Philips) and a field emission scanning electron microscope (S-4000, Hitachi). TGA was also performed by thermogravimetry- (TG)-DTA (NETZSCH, STA 409 PC).

Figures 2(a) and 2(b) show surface images of regenerated cellulose films under 120 and $180 \mathrm{~min}$ exposure time of the magnetic field, respectively. Since the cellulose has negative diamagnetic anisotropy, the direction of alignment for the cellulose nanofibers is perpendicular to the magnetic field direction. Figure 2(a) shows slight alignment of cellulose nanofibers perpendicular to the magnetic field direction when exposed for $120 \mathrm{~min}$. Figure 2(b) shows highly ordered and aligned cellulose fibers perpendicular to the magnetic field, as more time was provided to allow fiber orientation.

Figure 3 shows the FESEM images of cross sections taken along the [Fig. 3(a)] parallel and [Fig. 3(b)] the perpendicular directions with respect to the magnetic field direction, respectively. Since the cellulose nanofibers are aligned to the magnetic field direction, Fig. 3(a) shows the perpendicular arrangement of the cellulose nanofibers with

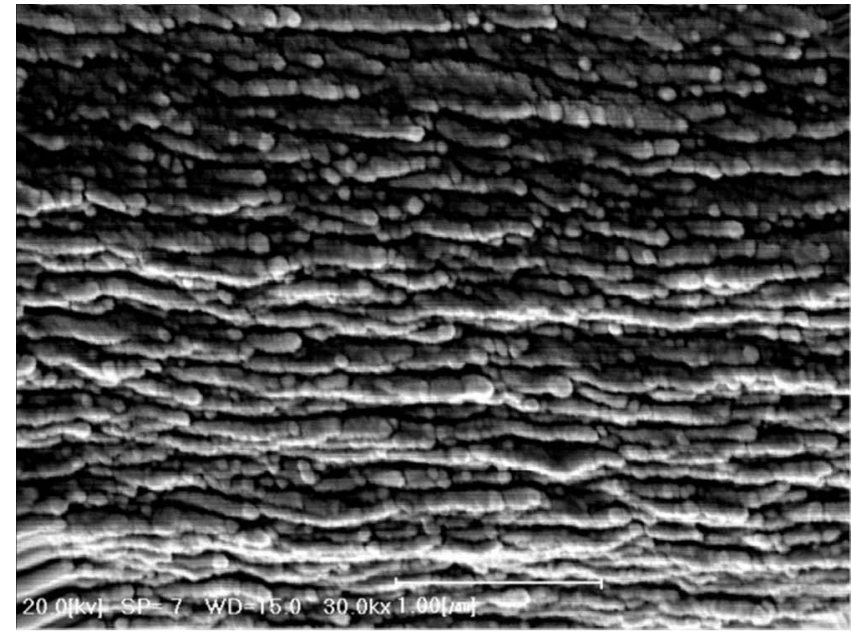

(a)

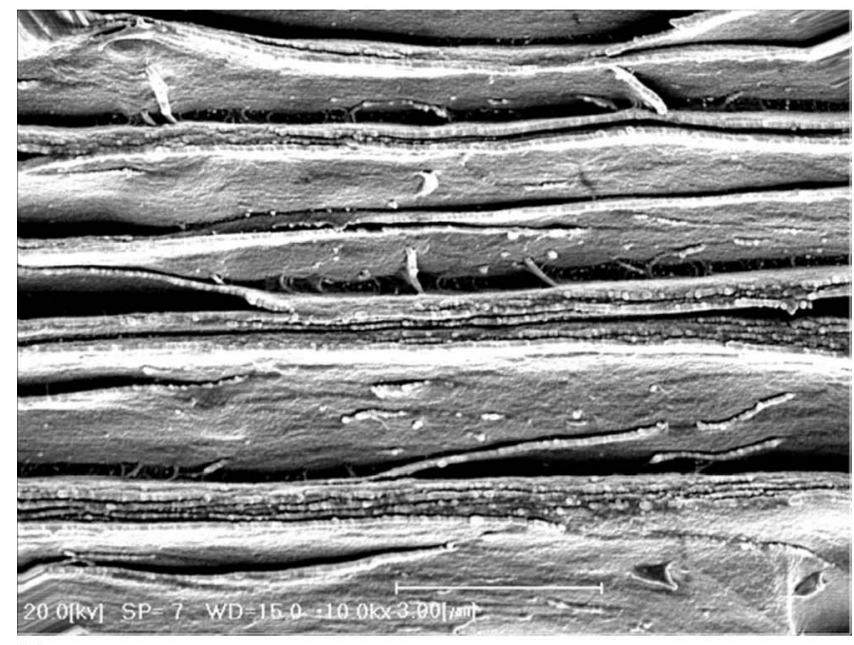

(b)

FIG. 3. FESEM views of cross sections taken along (a) the parallel and (b) the orthogonal with respective to the magnetic field direction.

respect to magnetic field direction. Figure 3(b) shows the layered and aligned cellulose structure parallel to the magnetic field direction.

Sun et $a .^{20}$ reported that purified cellulose had a higher thermal stability than the crude cellulose. The purified cellulose increased crystallinity so as to increase the thermal stability. The effect of cellulose nanofiber alignment induced by the magnetic field was investigated in terms of thermal behavior by taking TGA in the temperature range from room temperature to $600{ }^{\circ} \mathrm{C}$ at the rate of $10{ }^{\circ} \mathrm{C} / \mathrm{min}$ under nitrogen flow. Figure 4 shows the results. The decomposition temperatures were $263,275,278,283$, and $305{ }^{\circ} \mathrm{C}$ for the samples exposed to the magnetic field for $60,90,120,150$, and $180 \mathrm{~min}$, respectively. The decomposition temperature was increased as the duration of applied magnetic field increased. This may be due to the increased crystallinity ${ }^{20}$ by the applied magnetic field.

Figure 5(a) shows the x-ray diffraction profiles of regenerated cellulose under different magnetic field exposure times. These profiles were taken on the perpendicular direction with respect to the magnetic field on the regenerated cellulose. The definition of the (200) plane followed that of 


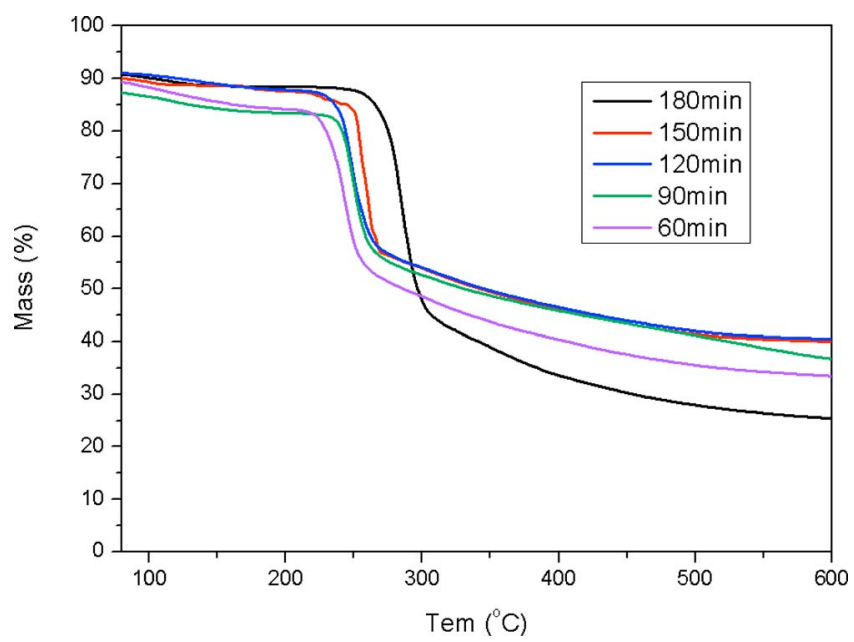

FIG. 4. (Color online) TGA graphs for the samples with different exposure times of the magnetic field.

Ref. 16. The (200) plane is parallel to the crystal $c$-axis. In the x-ray diffraction profiles two crystalline reflections at approximately $12^{\circ}$ and $20^{\circ}$ represent the (110) and (200) crystalline peaks. X-ray diffraction profiles of microcrystalline cellulose, regenerated cellulose, and regenerated cellulose reinforced using microcrystalline cellulose showed different crystalline structures. The microcrystalline part of the cotton cellulose is cellulose I, and the regenerated cellulose is cellulose II. ${ }^{21}$ Cellulose II has $12.1^{\circ}(110), 19.8^{\circ}(1 \tilde{10})$, and $22^{\circ}$ (200) peaks, while cellulose I has $14.8^{\circ}(110), 16.3^{\circ}(1 \tilde{10})$, and $22.6^{\circ}(200)$. The intensity at the $20.6^{\circ}$ peak of the regenerated cellulose increased without changing its position, as the duration of applied magnetic field increased. The crystalline peak representing (110) of the regenerated cellulose increased and shifted to $12.1^{\circ}, 12.8^{\circ}$, and $13.1^{\circ}$ as the exposure time of the magnetic field increased to 90,150 , and $180 \mathrm{~min}$, respectively. This information may not be sufficient to provide the crystal structure change since the change in peak intensity is small. Figure 5(b) shows the x-ray diffraction profiles of the sample along the perpendicular direction and the magnetic field direction when the exposure time is 180 min. Intensity peaks near $12^{\circ}$ and $20^{\circ}$ for the perpendicular direction case were higher than those of the magnetic field direction case. Thus, it is clear that this crystallinity increase is mainly due to the magnetic field.

In summary, the regenerated cellulose formed cellulose nanofibers and aligned perpendicularly to the magnetic field. Longer exposure time of magnetic field resulted better alignment of the nanofibers and increased decomposition temperature of the material. X-ray crystallogram results show that the crystallinity index increased as the exposure time increased.

This work is performed under the support of Creative Research Initiatives (EAPap Actuator) of KOSEF/MEST, South Korea.

${ }^{1}$ J. Chen, Q. Wang, Z. Hua, and G. Du, Enzyme Microb. Technol. 40, 1651 (2007).

${ }^{2}$ A. G. M. Scannell, C. Hill, R. P. Ross, S. Marx, W. Hartmeier, and E. K. Arendt, Int. J. Food Microbiol. 60, 241 (2000).

${ }^{3}$ A. T. Ram, Polym. Degrad. Stab. 29, 3 (1990).

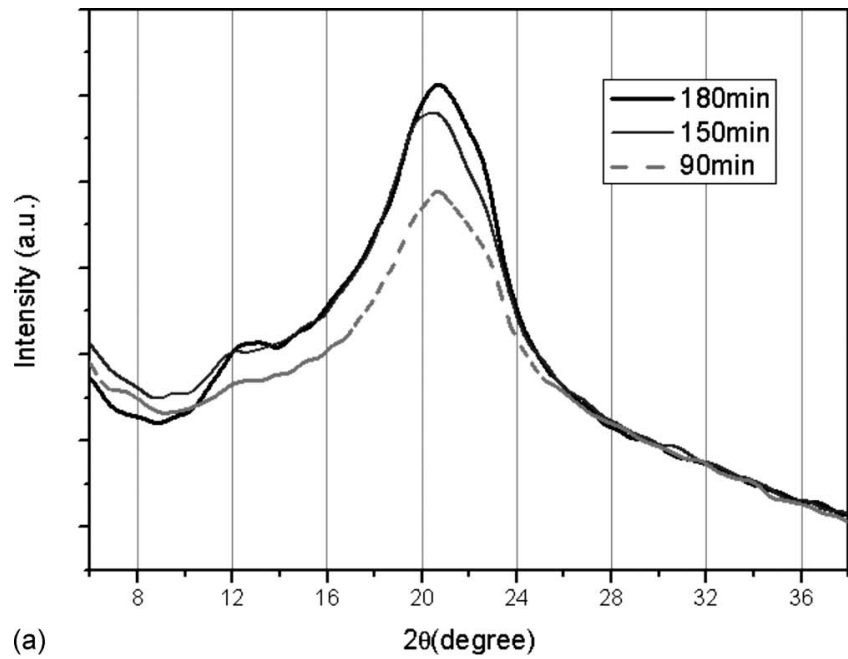

(a)

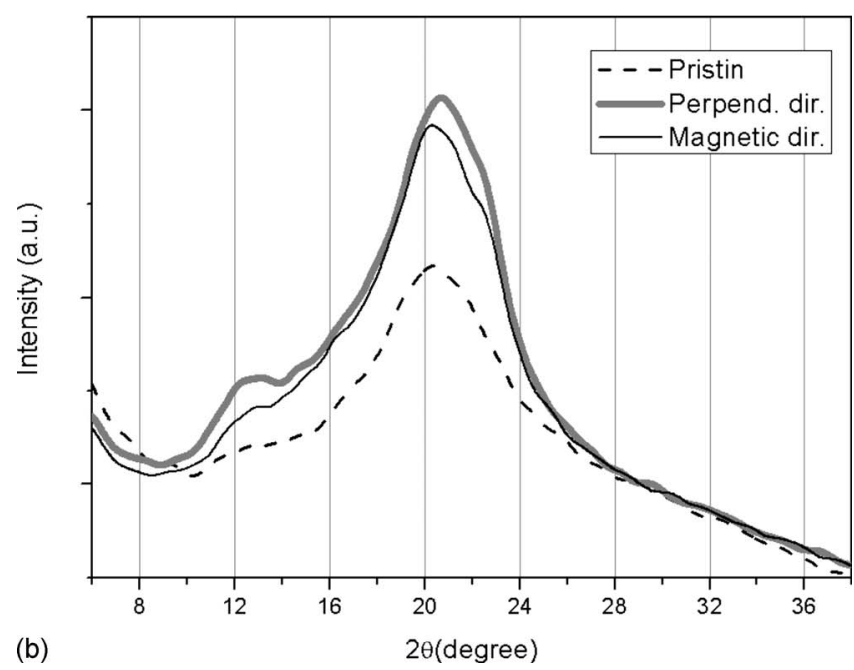

FIG. 5. X-ray crystallograms for (a) the samples with different exposure times of the magnetic field at the perpendicular direction, and (b) the sample when the exposure time is $180 \mathrm{~min}$ along the perpendicular and magnetic field directions as the pristine sample without magnetic field.

${ }^{4}$ F. Atyabi, E. Khodaverdi, and R. Dinarvand, Int. J. Pharm. 339, 213 (2007).

${ }^{5}$ J. Ganster, H.-H. Fink, and M. Pinnow, Composites, Part A 37, 1796 (2006).

${ }^{6}$ K. Y. Cho, H. K. Lim, Y. Chen, J. Kim, and K. S. Kang, Proc. SPIE 6528, 65280C (2007)

${ }^{7}$ X. Cai, B. Riedl, and A. Ait-Kadi, Composites, Part A 34, 1075 (2003).

${ }^{8}$ N. Reddy and Y. Yang, Polymer 46, 5494 (2005).

${ }^{9}$ E. Kristo and C. G. Biliaderis, Carbohydr. Polym. 68, 146 (2007).

${ }^{10}$ J. Kim, S. Yun, and Z. Ounaies, Macromolecules 39, 4202 (2006).

${ }^{11}$ J. Kim, C. S. Song, and S. R. Yun, Smart Mater. Struct. 15, 719 (2006).

${ }^{12}$ S. Y. Yun, J. Kim, and C. S. Song, Sens. Actuators, A 133, 225 (2007).

${ }^{13}$ A. N. Nakagaito, S. Iwamoto, and H. Yano, Appl. Phys. A: Mater. Sci. Process. 80, 93 (2005).

${ }^{14}$ W. Gindl, K. J. Martinschitz, P. Boesecke, and J. Keckes, Biomacromolecules 7, 3146 (2006).

${ }^{15}$ H. P. Fink, P. Weigel, and H. J. Gaster, Prog. Polym. Sci. 26, 1473 (2001).

${ }^{16}$ J. Sugiyama, H. Chanzy, and G. Maret, Macromolecules 25, 4232 (1992).

${ }^{17}$ E. D. Cranston and D. G. Gray, Sci. Technol. Adv. Mater. 7, 319 (2006).

${ }^{18}$ F. Kimura, T. Kumura, M. Tamura, A. Hirai, M. Ikuno, and F. Horii, Langmuir 21, 2034 (2005).

${ }^{19}$ T. Kimura, M. Yamato, S. Endo, F. Kimura, H. Hata, H. Kawasaki, and Y. Shinagawa, J. Polym. Sci., Part B: Polym. Phys. 39, 1942 (2001).

${ }^{20}$ J. X. Sun, F. Xu, X. F. Sun, B. Xiao, and R. C. Sun, Polym. Degrad. Stab. 88, 521 (2005).

${ }^{21}$ W. Gindl, K. J. Martinschitz, P. Boesecke, and J. Keckes, Compos. Sci. Technol. 66, 2639 (2006). 